\title{
Measurement of hot electron momentum relaxation times in metals by femtosecond ellipsometry
}

\author{
V. V. Kruglyak* and R. J. Hicken \\ School of Physics, University of Exeter, Exeter EX4 4QL, United Kingdom \\ M. Ali and B. J. Hickey \\ Department of Physics and Astronomy, University of Leeds, Leeds LS2 9JT, United Kingdom \\ A. T. G. Pym and B. K. Tanner \\ Department of Physics, University of Durham, Durham DH1 3LE, United Kingdom
}

(Received 28 February 2005; published 16 June 2005)

\begin{abstract}
Time-resolved ellipsometric measurements were made upon $\mathrm{Au}, \mathrm{Cu}, \mathrm{Ag}, \mathrm{Ni}, \mathrm{Pd}, \mathrm{Ti}, \mathrm{Zr}$, and $\mathrm{Hf}$ thin films. Using an elliptically polarized pump beam, the decay of the optically induced polarization of the sample was observed. Characteristic relaxation times are extracted and interpreted in terms of scattering of linear and angular momentum of hot electrons. A systematic variation is observed between different metals that reflects their underlying band structure.

DOI: 10.1103/PhysRevB.71.233104

PACS number(s): 78.47.+p, 42.65.-k, 78.20.Ls
\end{abstract}

Investigations of ultrafast electron dynamics in metals are required to understand and implement phenomena such as ultrafast demagnetization, ${ }^{1}$ ultrafast optical generation of magnons, ${ }^{2}$ phonons, ${ }^{3}$ and optical birefringence and dichroism. ${ }^{4}$ The time scales for the linear and angular momentum dynamics of electrons and holes are of particular importance for transport phenomena, where the carrier energy, direction of motion, and spin polarization play a significant role. ${ }^{5}$ The corresponding scattering times are determined by the interactions between electrons, holes, and phonons, and by scattering from defects, and are expected to be of the order of a few femtoseconds. ${ }^{6}$ Hence, there is a need for time-resolved (TR) experiments ${ }^{1-4,7}$ that have sufficient time resolution to resolve these processes.

The interaction between light and matter can be viewed as the creation of a coherent quantum superposition of initial and final electron states that has an associated polarization. Coherence between states with different wave vector requires an intermediate virtual state and the presence of a coherent phonon. A transition may occur when the coherence of the system is broken either due to the finite width of the optical wave packet or by scattering from the environment. The transition results in the absorption of a photon and the creation of a hot electron-hole pair. Values for the decoherence time have been deduced from electronic spectroscopic techniques and TR two-photon photoemission (TR 2PPE), although their physical interpretation is not straightforward. ${ }^{7,8}$ The polarization may persist for a longer time if the hot electron-hole pair retains some memory of the optically induced momentum after a scattering event.

TR optical measurements have been used to study the specular inverse Faraday effect (SIFE) and specular optical Kerr effect ${ }^{4,9}$ (SOKE) in metals, where it has always been assumed that the characteristic relaxation times are so short that the signal shape corresponds to the cross correlation of the pump and probe intensities. In this paper we show that pump-probe measurements of the incoherent part of the SIFE and SOKE may yield relaxation times for the transient polarization in metals that correspond to the relaxation times for the linear and angular momentum of the excited carriers. A correlation between the character of the TR polarization signal and the electronic structure of different groups of metals is revealed.

Measurements ${ }^{10}$ were performed with 90 fs (full width at half maximum, $\sigma)$ transform limited pulses $(\hbar \omega$ $=1.575 \pm 0.005 \mathrm{eV}$ ) from a Ti:sapphire laser with $80 \mathrm{MHz}$ repetition rate. The pump and probe were focused to a $15 \mu \mathrm{m}$ spot size, had energies of 2 and $0.04 \mathrm{~nJ}$, and were incident upon the sample at $7^{\circ}$ and $27^{\circ}$, respectively. The probe was $p$ polarized while the pump polarization was varied continuously from $p$ to a circularly polarized state by rotating a quarter-wave plate placed after a polarizer. The delay between the two pulses could be varied with a step size of $1.67 \mathrm{fs}$. Coherent contributions to the signal were suppressed by modulating the time delay through a few wavelengths. The pump was chopped and pump-induced changes in the intensity and polarization of the reflected probe were sensed simultaneously by an optical bridge. A quarter-wave plate was placed in front of the bridge detector to measure ellipticity $\eta$ rather than rotation $\theta$.

We studied thin films of noble $[\mathrm{Cu}(32.60 \pm 0.05 \mathrm{~nm}), \mathrm{Ag}$ $(24.2 \pm 0.3 \mathrm{~nm}), \mathrm{Au}(28.10 \pm 0.03 \mathrm{~nm})]$ and transition $[\mathrm{Ni}$ $(53.6 \pm 0.4 \mathrm{~nm}), \mathrm{Pd}(61.8 \pm 0.7 \mathrm{~nm}), \mathrm{Ti}(25.6 \pm 0.4 \mathrm{~nm}), \mathrm{Zr}$ $(42.50 \pm 0.07 \mathrm{~nm})$, Hf $(76.60 \pm 0.14 \mathrm{~nm})]$ metals, deposited by magnetron sputtering onto $\mathrm{Si}$ substrates. The thicknesses were determined by grazing incidence $\mathrm{x}$-ray reflectivity. The $\mathrm{Ni}$ film was magnetized perpendicular to the plane of incidence so that ultrafast demagnetization did not contribute to the polarization signal. ${ }^{11}$

The amplitudes of transient SIFE and SOKE may be related to the cubic components $\chi_{x x y y}$ and $\chi_{x y y x}$ of the nonlinear susceptibility tensor ${ }^{9,10}$ by

$$
\left(\begin{array}{c}
\Delta \theta \\
\Delta \eta
\end{array}\right)=\frac{1}{2} \sin 4 \varphi\left(\begin{array}{c}
\operatorname{Re} \\
\operatorname{Im}
\end{array}\right) F_{+}+\sin 2 \varphi\left(\begin{array}{c}
-\operatorname{Im} \\
\operatorname{Re}
\end{array}\right) F_{-},
$$

where 
(a)

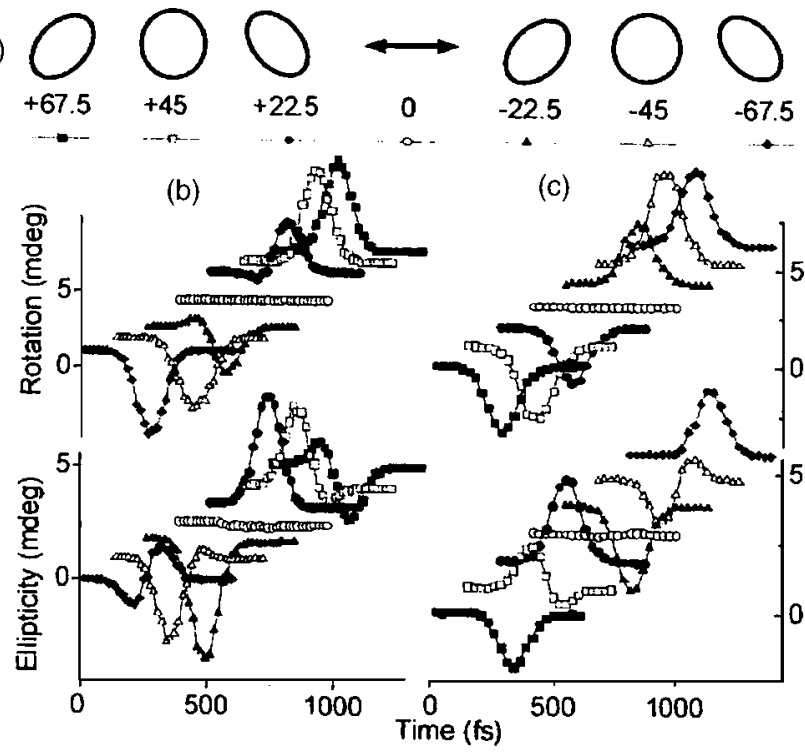

FIG. 1. (a) The pump polarization state for different orientations of the quarter-wave plate is shown. (b),(c) Rotation and ellipticity signals obtained for different orientations of the pump quarter-wave plate are shown for $\mathrm{Ti}$ and $\mathrm{Pd}$, respectively.

$$
F_{ \pm}=-\frac{32 \pi^{2} I_{\text {pump }}}{c|1+n|^{2}}\left\{\frac{\chi_{x x y y} \pm \chi_{x y y x}}{n\left(1-n^{2}\right)}\right\} \text {. }
$$

Here, $\varphi$ is the angle between the plane of incidence and the fast axis of the pump quarter-wave plate, $I_{\text {pump }}$ is the pump intensity, $n$ is the refractive index of the medium, and $c$ is the speed of light.

Examples of transient rotation and ellipticity signals for different $\varphi$ are shown in Fig. 1 for Ti and Pd. The signals consist of two components, the magnitudes of which vary with periods of $90^{\circ}$ and $180^{\circ}$ with respect to $\varphi$ as expected from Eq. (1). The period of $90^{\circ}$ is characteristic of the SOKE, a pump-induced linear birefringence associated with optical orientation of linear momentum. The SOKE increases as the ellipticity angle of the pump approaches $0^{\circ}$ or $90^{\circ}$, and as the angle between the major axis of the pump beam ellipse and the plane of probe polarization approaches $45^{\circ}$. The SOKE vanishes when the pump is either circularly polarized (e.g., for $\varphi= \pm 45^{\circ}$ ) or linearly polarized parallel or perpendicular to the probe (e.g., for $\varphi=0^{\circ}$ ). The period of $180^{\circ}$ is characteristic of the SIFE, a pump-induced circular birefringence associated with optical orientation of angular momentum. The SIFE has maximum amplitude for circular pump polarization (for $\varphi= \pm 45^{\circ}$ ), and vanishes when the pump is linearly polarized (for $\varphi=0,90^{\circ}$ ). The shape of the signals was found to be independent of the pump energy up to the measurement value of $2 \mathrm{~nJ}$, and the signal amplitude varied linearly with pump intensity which is characteristic of thirdorder nonlinearities such as SIFE and SOKE. ${ }^{9}$

From Eq. (1) the signals are expected to consist of a linear superposition of the SIFE and SOKE and so the two effects can be separated. For example, for pairs of signals with $\varphi$ equal to $+22.5^{\circ}$ and $+67.5^{\circ}$ (or $-22.5^{\circ}$ and $-67.5^{\circ}$ ) the SIFE component has the same magnitude and sign, while the

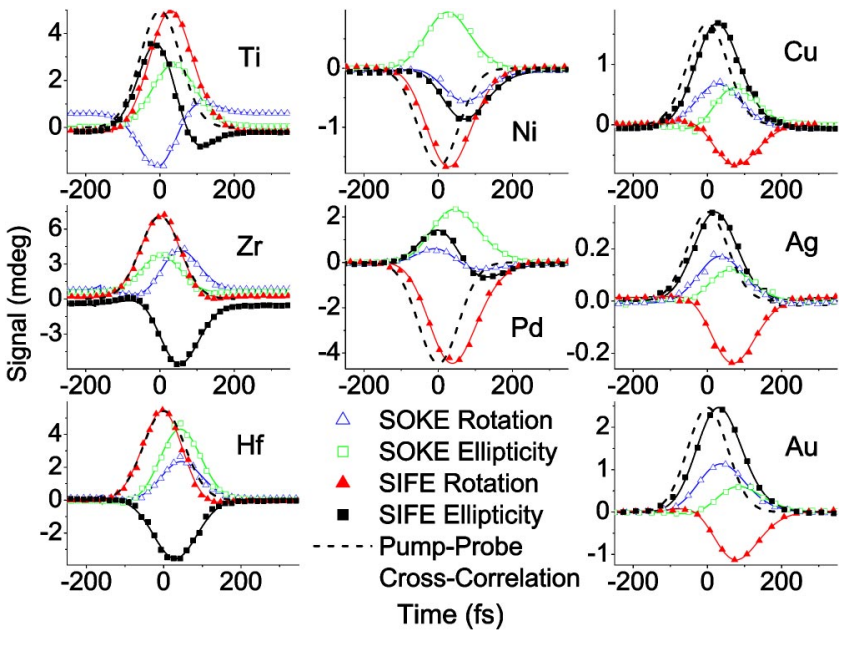

FIG. 2. (Color online) The SIFE and SOKE contributions to the transient polarization response [symbols are data and solid curves are fits to Eq. (2)] are shown with the pump-probe intensity cross correlation for the different samples measured.

SOKE has the same magnitude but opposite sign. ${ }^{10}$ Hence, the difference of the two signals divided by 2 contains only the SOKE component. Their sum contains only the SIFE component, but with amplitude greater by a factor of $\sqrt{2}$ in comparison with the pure SIFE signal measured at $\varphi$ $= \pm 45^{\circ}$. The latter equivalence was found to be accurately satisfied, justifying the procedure used to separate the SIFE and SOKE. The SOKE and SIFE contributions extracted by this method are plotted in Fig. 2 for different metals. ${ }^{12}$

We begin by noting the similarity of the relative signs and magnitudes of the SIFE and SOKE signals in Fig. 2 within the group of noble metals. $\mathrm{Zr}$ and Hf define a second group to which Ti can be assigned if one assumes that the bipolar shapes of the Ti SIFE ellipticity and SOKE rotation traces result from the presence of two peaklike contributions (two each for SIFE and SOKE) that have different center positions and opposite signs. One of these peaks tends to make the SIFE and SOKE signals similar to those of $\mathrm{Zr}$ and $\mathrm{Hf}$, while the other peak breaks the similarity. Making similar allowance for the bipolar shape of the Pd SIFE ellipticity and SOKE rotation signals, $\mathrm{Pd}$ and $\mathrm{Ni}$ form another group. The relative signs and magnitudes differ substantially between the different groups, which lie in different columns of the periodic table. The electronic structure is similar within each group as far as optical properties are concerned. For example, at our photon energy the optical response of the noble metals is due to intraband optical transitions, ${ }^{13}$ while that of $\mathrm{Ti}, \mathrm{Zr}$, and $\mathrm{Hf}$ is dominated by interband transitions. ${ }^{14}$ Both interband and intraband transitions can contribute to the optical response of $\mathrm{Ni}$ and $\mathrm{Pd} .^{15}$

For a particular metal, the maximum values of the SIFE and SOKE signals in Fig. 2 occur at different time delays in both rotation and ellipticity. ${ }^{16}$ Therefore the transient polarization signal cannot be described as a cross correlation of the pump and probe intensity, since the zero time delay position must be uniquely defined. Instead the shapes of the SIFE and SOKE signals must be described as a double convolution of the pump and probe profiles with the product of 
the Heaviside step function and an exponential function that contains the characteristic relaxation time of the transient polarization. ${ }^{17}$ As the value of the relaxation time increases, the TR signal broadens and becomes asymmetric, and the position of the maximum shifts toward positive time delay. ${ }^{18}$ These effects are hardly noticeable for relaxation times smaller than the pulse width $\sigma$. However, when both SIFE and SOKE are present in the same signal and characterized by different relaxation times, then the shift between their maximum values becomes apparent as the signal shape becomes bipolar. In addition, the bipolar shapes of the SIFE and SOKE components themselves extracted for Ti and Pd suggest that each effect may contain two contributions of opposite sign with different relaxation times. We therefore use the sum of two double convolutions to fit the data of Fig. 2:

$$
\begin{aligned}
& \theta^{\mathrm{SIFE}(\mathrm{SOKE})}\left(\eta^{\mathrm{SIFE}(\mathrm{SOKE})}\right) \propto \frac{1}{2} \sum_{j=1}^{2} A_{\theta(\eta), j}^{\mathrm{SIFE}(\mathrm{SOKE})} \\
& \quad \times\left[1+\operatorname{erf}\left(\frac{\left(t-t_{0}\right) \sqrt{2 \ln 2}}{\sigma}-\frac{\sigma}{2 \tau_{j}^{\mathrm{SIFE}(\mathrm{SOKE})} \sqrt{2 \ln 2}}\right)\right] \\
& \quad \times \exp \left\{-\frac{\left(t-t_{0}\right)}{\left.\tau_{j}^{\mathrm{SIFE}(\mathrm{SOKE})}\right\},}\right.
\end{aligned}
$$

where $A_{\theta(\eta), j}^{\mathrm{SIFE}(\mathrm{SOKE})}$ are the amplitudes of the signal components. For a particular metal, the same four relaxation times $\tau_{1}^{\mathrm{SIFE}(\mathrm{SOKE})}$ and $\tau_{2}^{\mathrm{SIFE}(\mathrm{SOKE})}$ were used in fitting SIFE and SOKE rotation and ellipticity signals, while the value of the zero delay time $t_{0}$ was the same for all four traces. It was not possible to fit the four traces for a particular metal with models that contained fewer relaxation times. In cases where the percentage difference in the two relaxation times for a particular effect was small (less than 10\%), the values of the two associated amplitudes were found to almost cancel and hence the errors in the fitted parameters were comparable to or larger than the parameter values. This suggests that a more accurate model or a shorter pulse width may be needed. Nevertheless, our simple model fits the observed signals well (Fig. 2) and reveals the characteristic time scales for the dynamics, summarized in Table I.

The values presented in Table I fall into two categories. In the first, occupied by the noble metals and $\mathrm{Ni}$, the difference of the two decay times for SIFE (or SOKE) is small. This suggests that the transient polarization response results from excitation of transitions in one particular region of the band structure. The initial and final states occupy finite phase volumes that may be large for intraband transitions. The corresponding relaxation time will generally exhibit dispersion associated with the range of initial and final states that are involved. The use of two similar relaxation times in our model provides a crude representation of this dispersion, which may lead to a small shift in the position of the maximum in the signal. Alternatively, if the relaxation times differ significantly (as in $\mathrm{Hf}, \mathrm{Zr}$, Pd SOKE, and Ti SIFE), the signal may involve states from two different regions of the band structure. These states may even have the same energy, since
TABLE I. The relaxation times obtained by fitting the SIFE and SOKE contributions shown in Fig. 2 to Eq. (2) are presented.

\begin{tabular}{lcccccc}
\hline \hline & \multicolumn{2}{c}{ SIFE } & & \multicolumn{2}{c}{ SOKE } \\
\cline { 2 - 3 } \cline { 5 - 6 } & $\tau_{1}(\mathrm{fs})$ & $\tau_{2}(\mathrm{fs})$ & & $\tau_{1}(\mathrm{fs})$ & $\tau_{2}(\mathrm{fs})$ \\
\hline $\mathrm{Cu}$ & 25 & 27.5 & & 22.5 & 23.5 \\
$\mathrm{Ag}$ & 24.5 & 26 & & 22 & 22.5 \\
$\mathrm{Au}$ & 27.5 & 28 & & 31.5 & 32 \\
$\mathrm{Ni}$ & 25.5 & 26.5 & & 24.5 & 25 \\
$\mathrm{Pd}$ & 30 & 31.5 & & 9.5 & 44 \\
$\mathrm{Ti}$ & 9 & 23 & & 17 & 18 \\
$\mathrm{Zr}$ & 10.5 & 14.5 & & 10 & 13 \\
$\mathrm{Hf}$ & 11 & 13 & & 10.5 & 12 \\
\hline \hline
\end{tabular}

TR 2PPE experiments ${ }^{7}$ have shown that hot electron lifetimes at a particular energy depend on whether excitation was caused by an interband or intraband transition. Note that the relaxation time values presented in Table I are in order of magnitude agreement with those obtained from calculations and measurements made using other techniques. ${ }^{6-8}$ However, a more accurate comparison seems infeasible due to the large scatter in the values presented in the references.

The action of a linearly polarized pump pulse reduces the symmetry of the momentum space electron distribution and induces an axial symmetry. The SOKE relaxation times characterize the recovery of the symmetry of the distribution, but only in the region of the band structure sensed by the probe beam. For interband transitions, the detection region is usually narrow. Therefore any inelastic scattering results in removal of an electron (hole) from the detection region and leads to a change in the measured linear momentum. For intraband transitions, the detection region may range continuously by up to one photon energy above and below the Fermi level. Inelastically scattered electrons and holes are then more likely to remain within the detection region and contribute to the transient polarization response. This implies that experiments involving intraband transitions should provide a better estimate of the momentum relaxation time. The action of a circularly polarized pulse leads to a repopulation of electrons and holes between states with different angular momentum component in the direction of the pump pulse wave vector. The corresponding SIFE relaxation time describes the recovery of the symmetry of the initial angular momentum distribution. Again it is preferred that the probe response is dominated by intraband transitions or else inelastic scattering may obscure the relaxation of hot electron (hole) angular momentum.

In summary, femtosecond ellipsometric measurements performed with an elliptically polarized pump have been made to directly investigate the ultrafast decay (with decay time shorter than the pulse duration) of optically induced transient linear and circular polarization in metals. The response of different metals has been shown to fall into groups that correspond to columns of the periodic table. A simple model has been introduced that fits the shapes of the signals. In some cases two distinct relaxation times may be identified, that are interpreted in terms of scattering of the linear 
and angular momentum of the excited electrons. These relaxation times may be relevant to transport in hot electron devices such as the spin valve transistor or magnetic tunnel transistor. The capability of this technique could be further enhanced through the use of shorter pulses and by tuning the wavelength of pump and probe, so that the mechanisms responsible for linear and angular momentum relaxation in metals may be explored and understood. Our results also emphasize the need for a more rigorous theory of the tran- sient third-order nonlinearity so that the underlying relaxation times can be more accurately determined.

The authors acknowledge the financial support of the U.K. Engineering and Physical Sciences Research Council (EPSRC), the New Energy and Industrial Technology Development Organization (NEDO), and the Overseas Research Student (ORS) scheme, and the assistance of R. Wilks and A. Barman in setting up the apparatus.
*Author to whom correspondence should be addressed. Email address: V.V.Kruglyak@exeter.ac.uk

${ }^{1}$ G. P. Zhang, W. Hübner, E. Beaurepaire, and J.-Y. Bigot, Top. Appl. Phys. 83, 245 (2001); B. Koopmans, ibid. 87, 253 (2003).

${ }^{2}$ M. van Kampen, C. Jozsa, J. T. Kohlhepp, P. LeClair, L. Lagae, W. J. M. de Jonge, and B Koopmans, Phys. Rev. Lett. 88, 227201 (2002); Q. Zhang, A. V. Nurmikko, A. Anguelouch, G. Xiao, and A. Gupta, ibid. 89, 177402 (2002).

${ }^{3}$ J. Hohlfeld, S. S. Wellershoff, J. Güdde, U. Conrad, V. Jahnke, and E. Matthias, Chem. Phys. 251, 237 (2000); U. Bovensiepen, A. Melnikov, I. Radu, O. Krupin, K. Starke, M. Wolf, and E. Matthias, Phys. Rev. B 69, 235417 (2004).

${ }^{4}$ N. I. Zheludev, P. J. Bennett, H. Loh, S. V. Popov, I. R. Shatwell, Y. P. Svirko, V. E. Gusev, V. F. Kamalov, and E. V. Slobodchikov, Opt. Lett. 20, 1368 (1995); P. J. Bennett, V. Albanis, Y. P. Svirko, and N. I. Zheludev, ibid. 24, 1373 (1999); V. V. Kruglyak, R. J. Hicken, M. Ali, B. J. Hickey, A. T. G. Pym, and B. K. Tanner, J. Opt. A, Pure Appl. Opt. 7, S235 (2005).

${ }^{5}$ R. Jansen, J. Phys. D 36, R289 (2003).

${ }^{6}$ R. Knorren, G. Bouzerar, and K. H. Bennemann, J. Phys.: Condens. Matter 14, R739 (2002).

${ }^{7}$ H. Petek and S. Ogawa, Prog. Surf. Sci. 56, 239 (1997).

${ }^{8}$ E. Knoesel, A. Hotzel, and M. Wolf, Phys. Rev. B 57, 12812 (1998); S. Link, H. A. Dürr, and W. Eberhardt, J. Phys.: Condens. Matter 13, 7873 (2001).

${ }^{9}$ S. V. Popov, Yu. P. Svirko, and N. I. Zheludev, J. Opt. Soc. Am. B 13, 2729 (1996); Y. P. Svirko and N. I. Zheludev, Polarization of Light in Nonlinear Optics (Wiley, New York, 1998).

${ }^{10}$ R. Wilks, N. D. Hughes, and R. J. Hicken, J. Phys.: Condens.
Matter 15, 5129 (2003).

${ }^{11}$ T. Kampfrath, R. G. Ulbrich, F. Leuenberger, M. Munzenberg, B. Sass, and W. Felsch, Phys. Rev. B 65, 104429 (2002).

${ }^{12}$ Measurements were also performed with a linearly polarized pump of variable orientation so that only the SOKE contributed to the signal (not shown). The measured SOKE components were found to agree very well both in shape and value with those shown in Fig. 2.

${ }^{13}$ H. Eckardt, L. Fritsche, and J. Noffke, J. Phys. F: Met. Phys. 14, 97 (1984).

${ }^{14}$ D. W. Lynch, C. G. Olson, and J. H. Weaver, Phys. Rev. B 11, 3617 (1975).

${ }^{15}$ P. B. Johnson and R. W. Christy, Phys. Rev. B 9, 5056 (1974); J. H. Weaver, ibid. 11, 1416 (1975).

${ }^{16}$ To remove any ambiguity in the comparison of rotation and ellipticity signals, the zero-time-delay position was defined from the reflectivity signals that were recorded simultaneously.

${ }^{17} \mathrm{We}$ adopt this idea from TR 2PPE studies where the signal was fitted to a convolution of a symmetric exponential function with a pulse shape (see page 270 of Ref. 7). A. V. Kimel, F. Bentivegna, V. N. Gridnev, V. V. Pavlov, R. V. Pisarev, and T. Rasing, Phys. Rev. B 63, 235201 (2001) used a function similar to ours, but containing in addition a term equal to a simple convolution of the pump and probe intensities, to describe the transient polarization in GaAs.

${ }^{18}$ In our analysis, we have assumed a Gaussian pulse shape for the laser pulse. Simulations performed with the hyperbolic secant pulse shape have shown that the observed effects are qualitatively the same for both pulse shapes, while the corresponding relaxation times may differ by about $20 \%$. 